\title{
Combining Nelfinavir With Chloroquine Inhibits In Vivo Growth of Human Lung Cancer Xenograft Tumors
}

\author{
JACLYN LOPICCOLO ${ }^{1}$, SHIGERU KAWABATA ${ }^{2 *}$, JOELL J. GILLS ${ }^{2}$ and PHILLIP A. DENNIS ${ }^{2 * *}$ \\ ${ }^{1}$ Medical Oncology Branch, Center for Cancer Research, National Cancer Institute, Bethesda, MD, U.S.A.; \\ ${ }^{2}$ Department of Oncology, Johns Hopkins University School of Medicine, Baltimore, MD, U.S.A.
}

\begin{abstract}
Background/Aim: Nelfinavir is a human immunodeficiency virus protease inhibitor that is currently being repositioned as an anticancer drug. Chloroquine, an anti-malarial lysosomotropic drug, inhibits autophagy. It has been reported that the combination of nelfinavir and chloroquine significantly enhances endoplasmic reticulum (ER) stress and induces selective cell death in multiple cell line models (in vitro). Materials and Methods: We assessed the effects of the combination of these drugs on human NSCLC cell lines in vitro using cell proliferation assay and performed preclinical treatment studies using cell line-derived xenograft mouse models in vivo. Results: In vitro, this combination enhanced inhibition of NSCLC cell proliferation with increased proteotoxicity, including ER stress, and apoptosis. In vivo, the growth of human NSCLC xenograft tumors was inhibited, which correlated with increased apoptosis and induction of ER stress as well as NSCLC growth in vitro. Conclusion: Our findings suggest that the induction of proteotoxicity provides a promising new target for developing anticancer drugs.
\end{abstract}

Nelfinavir is a human immunodeficiency virus protease inhibitor that is currently being repositioned as an anticancer drug, in a process that is referred to as 'drug repurposing'

This article is freely accessible online.

*Current address: Department of Pathology, Osaka Medical College Faculty of Medicine, Takatsuki City, Osaka, Japan.

**Current address: Global Clinical Lead, Lung Cancer ImmunoOncology, AstraZeneca, Gaithersburg, MD, U.S.A.

Correspondence to: Shigeru Kawabata, Department of Pathology, Osaka Medical College Faculty of Medicine, 2-7 Daigakumachi, Bldg. C01, Fifth Floor, Takatsuki City, Osaka 569-8686, Japan. Tel: +81 726831221 (Ext. 8529), Fax: +81 726846513, e-mail: pa1030@osaka-med.ac.jp

Key Words: Nelfinavir, chloroquine, proteotoxicity, non-small cell lung cancer.
(1). Our group showed that nelfinavir induces endoplasmic reticulum (ER) stress due to proteotoxicity and apoptosis, and inhibits the proliferation of many types of cancer cells, including non-small cell lung cancer (NSCLC) cells (2). Additionally, we reported that combining nelfinavir with bortezomib, a proteasome inhibitor, enhances proteotoxicity in NSCLC and multiple myeloma (MM) cells in vitro and in vivo (3).

Chloroquine, an anti-malarial lysosomotropic drug, inhibits autophagy by increasing lysosomal $\mathrm{pH}$ and preventing autophagosome-lysosome fusion (4). Chloroquine also represents a prominent case of drug repurposing for cancer therapy against glioblastoma, MM, breast cancer, pancreatic cancer, etc. (5). Johnson et al. reported that the combination of nelfinavir and chloroquine significantly increases ER stress and induces selective cell death in multiple cell line models (in vitro) with hyperactive mammalian/mechanistic target of rapamycin complex 1, including human NSCLC H460 cells, in an autophagyindependent manner (6). This finding led us to hypothesize that combining nelfinavir with chloroquine might inhibit NSCLC tumor growth in vivo. To test our hypothesis, we assessed the effects of their combination on human NSCLC cell lines in vitro and on cell line-derived xenograft mouse models. Our data demonstrate the proteotoxic effects of combining nelfinavir with chloroquine in vitro and in vivo.

\section{Materials and Methods}

Cell culture. H157 and A549 NSCLC cell lines were obtained from the National Cancer Institute (NCI)/Navy Medical Oncology (Bethesda, MD, USA) and the American Type Culture Collection (Manassas, VA, USA), respectively, and maintained as described previously (2).

Reagents. Nelfinavir was obtained from the National Institutes of Health (NIH) AIDS reagent repository (Bethesda, MD, USA) and Pfizer Inc. (New York, NY, USA). Chloroquine was purchased from MP Biomedicals, Inc. (Solon, OH, USA). Primary antibodies for ubiquitin, cleaved/total poly ADP-ribose polymerase (PARP), and phospho (p)- eukaryotic initiation factor $2 \alpha$ (eIF2 $\alpha$ ) (Ser51) were 
purchased from Cell Signaling Technology (Beverly, MA, USA). Activating transcription factor 3 (ATF3) (C-19) antibody was purchased from Santa Cruz Biotechnology (Santa Cruz, CA, USA). Antibody to microtubule-associated protein 1A/1B-light chain 3 (LC-3) (2G6) was purchased from NanoTools (Teningen, Germany). Antibodies against actin and tubulin were purchased from Calbiochem (EMD Chemicals Inc. Gibbstown, NJ, USA) and Sigma (St. Louis, MO, USA), respectively.

Cell proliferation assay. $\mathrm{H} 157$ and A549 cells (2,500 cells per well) were plated in 96-well plates and treated with the following drug alone or in simultaneous combination for $48 \mathrm{~h}$ at the increasing concentrations either between $0.625 \mu \mathrm{M}$ and $10 \mu \mathrm{M}$ for nelfinavir dissolved in dimethyl sulfoxide (DMSO) or between $5 \mu \mathrm{M}$ and 80 $\mu \mathrm{M}$ for chloroquine dissolved in phosphate-buffered saline. Growth inhibition was determined as described previously (7). Experiments were performed three times, and each drug concentration was evaluated in sextuplet wells for a given experiment.

Immunoblotting analysis. Cells $\left(5 \times 10^{5}\right.$ cells per well) were plated in 6well plates and treated with either DMSO, $10 \mu \mathrm{M}$ nelfinavir, $80 \mu \mathrm{M}$ chloroquine, or their combination for $0.5,8,24$, or $48 \mathrm{~h}$ in timedependent manner and lysed in $2 \times$ lysis buffers as described previously (7). For tumor-tissue homogenates in vivo, frozen tumors were prepared as also described previously (7). Cell lysates or tumor-tissue homogenates with equal amounts of protein were separated using sodium dodecyl sulphate-polyacrylamide gel electrophoresis and then transferred to nitrocellulose membranes. Immunoblotting analyses were performed as described previously (7) and repeated at least three times. Immunoblot experiments were performed at least three times.

Drug treatment in vivo. Cells $\left(5 \times 10^{6} \mathrm{H} 157\right.$ or $\left.\mathrm{H} 549\right)$ in $100 \mu \mathrm{l}$ phosphate-buffered saline were subcutaneously injected into both rear flanks of 6-week-old female athymic NCr-nu/nu mice (Charles River Labs, Frederick, MD, USA). When the transplanted tumors reached a volume of $50 \mathrm{~mm}^{3}$, the mice were divided into the following four groups (5 mice per group): (i) Vehicle (4\% DMSO, $5 \%$ polyethylene glycol, $5 \%$ Tween 80 in saline); (ii) $50 \mathrm{mg} / \mathrm{kg}$ nelfinavir; (iii) 50 $\mathrm{mg} / \mathrm{kg}$ chloroquine; or (iv) the combination of nelfinavir and chloroquine for intraperitoneal injection once daily. Animal weights and tumor sizes were measured every other day. In all studies, tumor volume was calculated using the formula $\mathrm{v}=\left(a b^{2}\right) / 2$, where $\mathrm{a}$ and $\mathrm{b}$ are the long axis and the short axis, respectively. Immunoblotting analysis was performed for markers of apoptosis and ER stress. Densitometry was performed using ImageJ version 1.52 software (8). To obtain the levels of each marker, normalization to tubulin for each sample was performed. In vivo experiments were performed using a protocol approved by the NCI Animal Care and Use Committee (Animal Study Proposal Number: CTB-008).

Statistics. Statistical significance was analyzed using one-way ANOVA, and multiple comparisons were then performed using Dunn's test. All analyses were performed using GraphPad Prism software version 9 (GraphPad Software, Inc. San Diego, CA, USA). Statistical significance was set at $p<0.05$.

\section{Results}

Combining nelfinavir with chloroquine enhances NSCLC growth inhibition and proteotoxicity. To assess their effects on cellular proliferation, nelfinavir and chloroquine were tested. The combination of nelfinavir and chloroquine reduced proliferation more than either drug alone in both H157 and A549 cells (Figure 1A). To investigate the mechanism of growth inhibition, PARP cleavage was assessed by immunoblotting analysis (Figure 1B). The combination induced greater PARP cleavage than either drug alone, suggesting apoptotic cell death.

Moreover, to assess whether the combination enhanced proteotoxicity, we evaluated ubiquitinated proteins. Our findings showed that the combination enhanced the level of ubiquitin more than either drug alone, and the results represent proteotoxicity in the cytosol (Figure 1B). Additionally, the expression of ATF3, an ER stress marker, also increased more with the combination than with either drug alone, and this was evident by $24 \mathrm{~h}$. LC-3 II expression, a marker for autophagosomes, was not enhanced in the combination treatment compared with chloroquine alone. This finding suggests that nelfinavir and chloroquine do not affect autophagy in these NSCLC cells. Taken together, these data show that combining nelfinavir with chloroquine enhances proteotoxicity in a time-dependent manner in two different cell lines.

Combining nelfinavir with chloroquine inhibits NSCLC tumor growth in vivo. To determine whether the combination of nelfinavir and chloroquine enhanced the antitumor effects of either drug alone, we treated athymic $\mathrm{NCr}-\mathrm{nu} / \mathrm{nu}$ mice bearing $\mathrm{H} 157$ or A549 xenograft tumors with $50 \mathrm{mg} / \mathrm{kg}$ nelfinavir, $50 \mathrm{mg} / \mathrm{kg}$ chloroquine, or their combination. The combination of nelfinavir and chloroquine was well-tolerated and reduced tumor growth of $\mathrm{H} 157$ by nearly $75 \%$ (Figure 2A, upper panel) and of A549 by nearly $85 \%$ (Figure 2A, lower panel, and 2B) compared with the mice treated with vehicle. To evaluate the correlation between the antitumor effects and the mechanisms identified in vitro, markers of apoptosis and ER stress were evaluated in the tumors. The combination significantly increased the expression of cleaved PARP compared with the control in H157 xenograft tumors (Figure 2C). Because ATF3 did not increase in the group treated with the combination, we evaluated p-eIF2 $\alpha$, another ER stress marker. The combination significantly increased the expression of p-eIF $2 \alpha$ compared with that in the control. Taken together, these findings suggest that the combination inhibits tumor growth of NSCLC and enhances apoptosis induced by ER stress in vivo.

\section{Discussion}

Our data show that combining nelfinavir with chloroquine enhances proteotoxicity and inhibits in vivo growth of human NSCLC xenograft tumors as well as in vitro growth of NSCLC cells, which supports our hypothesis. Previously, we 

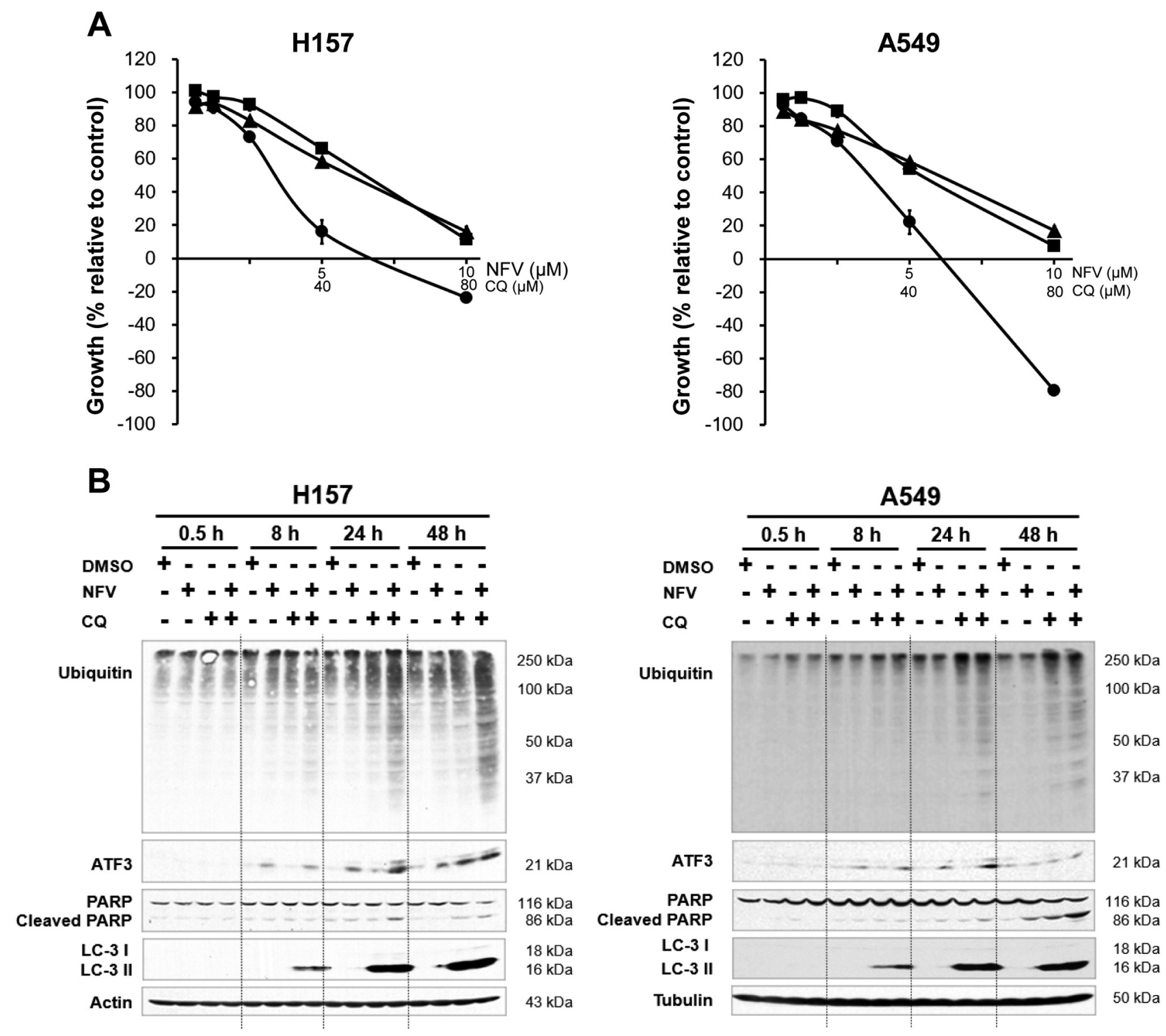

Figure 1. Combining nelfinavir (NFV) with chloroquine (CQ) enhances inhibition of non-small cell lung cancer (NSCLC) proliferation and proteotoxicity. A: Growth inhibition by nelfinavir $(\mathbf{\square})$, chloroquine $(\mathbf{\Delta})$, and their combination $(\mathbf{\bullet})$. H157 and A549 cells were treated with drugs at the indicated concentrations for 48 h. Data are the mean \pm SD. B: Enhancement of proteotoxicity. $H 157$ and A549 NSCLC cells were treated with either dimethyl sulfoxide (DMSO), $10 \mu \mathrm{M}$ nelfinavir, $80 \mu \mathrm{M}$ chloroquine, or their combination for the indicated times. Immunoblotting analysis was performed for markers of proteotoxicity, endoplasmic reticulum stress, apoptosis, and autophagy.

reported that combining nelfinavir with bortezomib enhances the disruption of protein homeostatic balance as a proteotoxic effect in NSCLC and MM cells in vitro and in vivo (3), suggesting the importance of proteotoxicity as a new target for developing anticancer drugs. In this study, we reconfirmed that nelfinavir can enhance proteotoxic effects when combined with drugs that induce proteotoxicity, such as chloroquine. These findings support our previous phase I clinical trial of nelfinavir as an anticancer agent for advanced solid tumors, including NSCLC (9). Taken together, the combination of nelfinavir and chloroquine may be an effective clinical strategy to maximize patient response to nelfinavir.

To our knowledge, this is the first study to describe in vivo growth inhibition of human NSCLC xenograft tumors by combining nelfinavir with chloroquine. Johnson et al. reported the efficacy of this combination in the H460 cell 


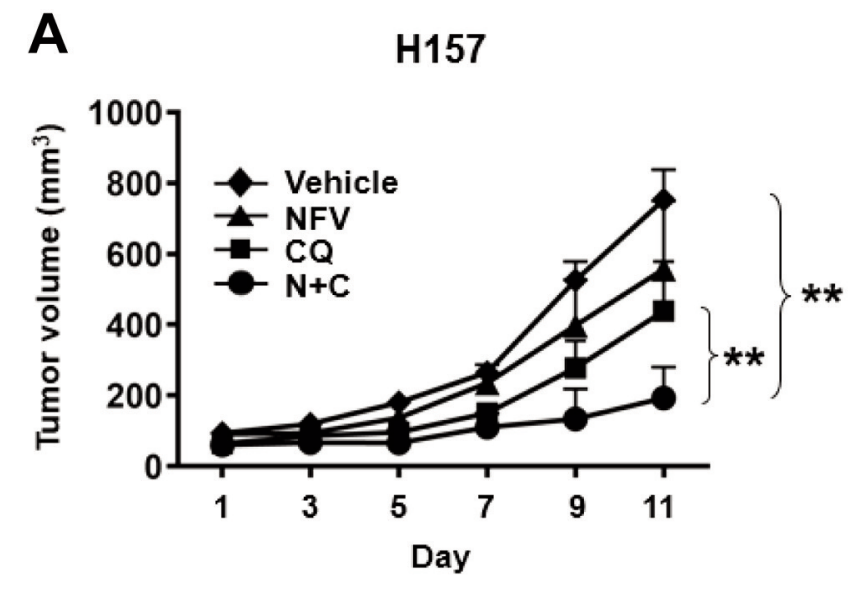

B
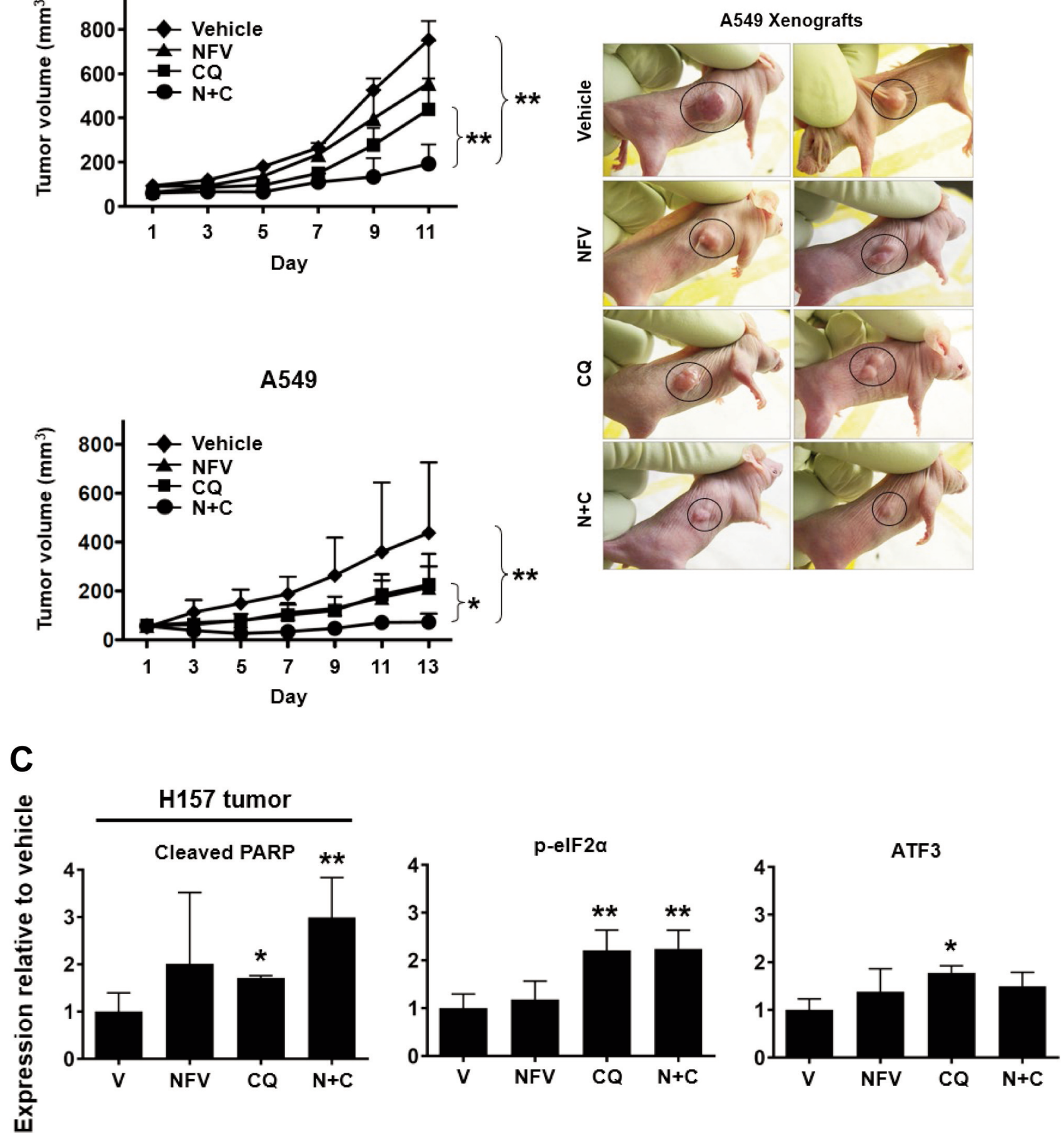

Figure 2. Combining nelfinavir (NFV) with chloroquine (CQ) inhibits tumor growth of non-small cell lung cancer (NSCLC) in vivo. A: Tumor growth of $H 157$ (upper panel) and A549 (lower panel) NSCLC cells as xenografts in athymic NCr-nu/nu. Data are the mean $\pm S D$. B: Representative photographs of two different mice bearing A549 xenograft tumors from each group after treatment for 13 days. Circles indicate tumors. C: Apoptosis and endoplasmic reticulum stress markers in vivo. The indicated markers were evaluated in the tumors using immunoblotting analysis as described in the Materials and Methods. V: Vehicle, $N+C$ : nelfinavir + chloroquine. Data are the mean $\pm S D$ of five mice. Significantly different at $* p<0.05$ and $* * 00.01$ compared with vehicle treatment. 
line in an in vitro study (6). In this study, we utilized two other NSCLC cell lines: H157 cells with KRAS (Q12R) and phosphatase and tensin homolog (PTEN, G251C) mutations (7), and A549 cells with a KRAS (Q12S) mutation (10). We also expanded our in vitro findings to an in vivo study. Our data show that combining nelfinavir with chloroquine enhances growth inhibition of $\mathrm{H} 157$ and A549 cells in vitro and in vivo, supporting the findings of Johnson et al. (6).

Autophagy plays a compensatory role in drug-induced proteotoxicity by activating autophagic degradation of misfolded proteins that results in harmful cellular effects (11). Chloroquine inhibits lysosome fusion with autophagosomes, resulting in faults in late autophagy and increased LC-3 II. Therefore, we expected that in combining nelfinavir with chloroquine, autophagy would be inhibited and result in enhanced proteotoxicity, compared with treatment with either drug alone. However, LC-3 II expression following combination treatment was similar to that following chloroquine administration alone (Figure 1B). This finding suggests that the proteotoxic mechanisms that result from combining nelfinavir with chloroquine might proceed in an autophagy-independent manner, as described by Johnson et al. (6).

In conclusion, we demonstrated that combining nelfinavir with chloroquine enhances inhibition of NSCLC growth and proteotoxicity in vitro and in vivo. Our findings suggest that the induction of proteotoxicity provides a promising new target for cancer therapy.

\section{Conflicts of Interest}

The Authors report no financial or other interests that could be construed as conflicts of interest.

\section{Authors' Contributions}

Conceived and designed the experiments: J.L., S.K., J.J.G. and P.A.D. Performed the experiments: J.L. and S.K. Analyzed the data: J.L., S.K. and J.J.G. Wrote the article: S.K. and P.A.D.

\section{Acknowledgements}

The Authors would like to thank Gail McMullen for assistance with the animal study, the NIH AIDS reagent repository, and Pfizer Inc. for the gift of nelfinavir. This work was supported by the Intramural Research Program of the Center for Cancer Research, NCI to P.A.D., and JSPS KAKENHI Grant Number JP20K07646 to S. K.

\section{References}

1 Maksimovic-Ivanic D, Fagone P, McCubrey J, Bendtzen K, Mijatovic S and Nicoletti F: HIV-protease inhibitors for the treatment of cancer: Repositioning HIV protease inhibitors while developing more potent NO-hybridized derivatives? Int J Cancer 140: 1713-1726, 2017. PMID: 27870005. DOI: 10.1002/ijc.30529
2 Gills JJ, LoPiccolo J, Tsurutani J, Shoemaker RH, Best CJM, Abu-Asab MS, Borojerdi J, Warfel NA, Gardner ER, Danish M, Hollander MC, Kawabata S, Tsokos M, Figg WD, Steeg PS and Dennis PA: Nelfinavir, a lead HIV protease inhibitor, is a broadspectrum, anticancer agent that induces endoplasmic reticulum stress, autophagy, and apoptosis in vitro and in vivo. Clin Cancer Res 13: 5183-5194, 2007. PMID: 17785575. DOI: 10.1158/10780432.CCR-07-0161

3 Kawabata S, Gills JJ, Mercado-Matos JR, LoPiccolo J, Wilson W, Hollander MC and Dennis PA: Synergistic effects of nelfinavir and bortezomib on proteotoxic death of NSCLC and multiple myeloma cells. Cell Death Dis 3: e353-12, 2012. PMID: 22825471. DOI: 10.1038/cddis.2012.87

4 Varisli L, Cen O and Vlahopoulos S: Dissecting pharmacological effects of chloroquine in cancer treatment: Interference with inflammatory signaling pathways. Immunology 159: 257-278, 2020. PMID: 31782148. DOI: 10.1111/imm.13160

5 Baker NC, Ekins S, Williams AJ and Tropsha A: A bibliometric review of drug repurposing. Drug Discov Today 23: 661-672, 2018. PMID: 29330123. DOI: 10.1016/j.drudis.2018.01.018

6 Johnson CE, Hunt DK, Wiltshire M, Herbert TP, Sampson JR, Errington RJ, Davies DM and Tee AR: Endoplasmic reticulum stress and cell death in mTORC1-overactive cells is induced by nelfinavir andenhanced by chloroquine. Mol Oncol 9: 675-688, 2015. PMID: 25498902. DOI: 10.1016/j.molonc.2014.11.005

7 Kawabata S, Chiang C Te, Tsurutani J, Shiga H, Arwood ML, Komiya T, Gills JJ, Memmott RM and Dennis PA: Rapamycin downregulates thymidylate synthase and potentiates the activity of pemetrexed in non-small cell lung cancer. Oncotarget 5: 10621070, 2014. DOI: 10.18632/oncotarget.1760

8 Schneider CA, Rasband WS and Eliceiri KW: NIH Image to ImageJ: 25 Years of image analysis. Nat Methods 9: 671-675, 2012. PMID: 22930834. DOI: 10.1038/nmeth.2089

9 Blumenthal GM, Gills JJ, Ballas MS, Bernstein WB, Komiya T, Dechowdhury R, Morrow B, Root H, Chun G, Helsabeck C, Steinberg SM, LoPiccolo J, Kawabata S, Gardner ER, Figg WD and Dennis PA: A phase I trial of the HIV protease inhibitor nelfinavir in adults with solid tumors. Oncotarget 5, 2014. PMID: 25327558. DOI: 10.18632/oncotarget.2415

10 Moran DM, Trusk PB, Pry K, Paz K, Sidransky D and Bacus SS: KRAS mutation status is associated with enhanced dependency on folate metabolism pathways in non-small cell lung cancer cells. Mol Cancer Ther 13: 1611-1624, 2014. PMID: 24688052. DOI: 10.1158/1535-7163.MCT-13-0649

11 Dikic I and Elazar Z: Mechanism and medical implications of mammalian autophagy. Nat Rev Mol Cell Biol 19: 349-364, 2018. PMID: 29618831. DOI: 10.1038/s41580-018-0003-4
Received November 6, 2020

Revised November 26, 2020

Accepted November 27, 2020 